\title{
Quality of life study of patients with unresectable locally advanced or metastatic pancreatic adenocarcinoma treated with gemcitabine+nab-paclitaxel versus gemcitabine alone: AX-PANC-SY001, a randomized phase-2 study
}

Suayib Yalcin ${ }^{1,2^{*}}$, Faysal Dane ${ }^{3}$, Berna Oksuzoglu ${ }^{4}$, Nuriye Yildirim Ozdemir ${ }^{5}$, Abdurrahman Isikdogan ${ }^{6}$, Metin Ozkann ${ }^{7}$, Guzin Gonullu Demirag ${ }^{8}$, Hasan Senol Coskun ${ }^{9}$, Bulent Karabulut ${ }^{10}$, Turkkan Evrensel ${ }^{11}$, Mehmet Ali Ustaoglu ${ }^{12}$, Feyyaz Ozdemir ${ }^{13}$, Hande Turna ${ }^{14}$, Tugba Yavuzsen ${ }^{15}$, Faruk Aykan ${ }^{16}$, Alper Sevinc ${ }^{17}$, Hakan Akbulut $^{18}$, Deniz Yuce ${ }^{2}$, Mutlu Hayran ${ }^{2}$ and Saadettin Kilickap ${ }^{2}$

\footnotetext{
Abstract

Background: Combination of gemcitabine and nab-paclitaxel has superior clinical efficacy than gemcitabine alone. Nevertheless, health-related quality of life. (QoL) associated with this combination therapy when administered at first-line in advanced pancreatic adenocarcinoma is unknown.

Methods: A total of 125 patients were randomized to combination therapy $(1000 \mathrm{mg} / \mathrm{m} 2$ gemcitabine $+125 \mathrm{mg} / \mathrm{m} 2$ nab-paclitaxel) and single-agent gemcitabine $(1000 \mathrm{mg} / \mathrm{m} 2)$ arms to take treatment weekly for 7 of 8 weeks, and following 3 of 4 weeks, until progression or severe toxicity. Primary endpoints were three-months of definitive deterioration free percent of patients, and QoL.

Results: Overall QoL analyses showed that 34 and $58.3 \%$ of cases in gemcitabine and gemcitabine+nab-P arms had no deterioration in 3rd month QoL scores $(p=0.018)$. These proportions were 27.3 and $36.6 \%$ in $6^{\text {th }}$ month assessments, respectively $(p=0.357)$. Median overall survivals in combination and single-agent arms were 9.92 months and 5.95 months, respectively (HR: $0.64,95 \% \mathrm{Cl}: 0.42-0.86, p=0.038$ ). Median progression free survivals in these treatment arms were 6.28 and 3.22 months, respectively (HR: $0.58,95 \% \mathrm{Cl}: 0.39-0.87, p=0.008$ ). Median time-to-deterioration were 5.36 vs 3.68 months, and objective response rates were $37.1 \%$ vs $23.7 \%(p=0.009)$, respectively in combination and single-agent arms.

(Continued on next page)
}

\footnotetext{
* Correspondence: suayibyalcin@gmail.com

${ }^{1}$ Hacettepe University Faculty of Medicine, Ankara, Turkey

${ }^{2}$ Hacettepe University Cancer Institute, Ankara, Turkey

Full list of author information is available at the end of the article
}

(c) The Author(s). 2020 Open Access This article is licensed under a Creative Commons Attribution 4.0 International License, which permits use, sharing, adaptation, distribution and reproduction in any medium or format, as long as you give appropriate credit to the original author(s) and the source, provide a link to the Creative Commons licence, and indicate if changes were made. The images or other third party material in this article are included in the article's Creative Commons licence, unless indicated otherwise in a credit line to the material. If material is not included in the article's Creative Commons licence and your intended use is not permitted by statutory regulation or exceeds the permitted use, you will need to obtain permission directly from the copyright holder. To view a copy of this licence, visit http://creativecommons.org/licenses/by/4.0/. The Creative Commons Public Domain Dedication waiver (http://creativecommons.org/publicdomain/zero/1.0/) applies to the data made available in this article, unless otherwise stated in a credit line to the data. 
(Continued from previous page)

Conclusions: Combination therapy with gemcitabine + nab-paclitaxel had better overall and progression-free survival than gemcitabine alone. Also, combination therapy showed increased response rate without toxicity or deteriorated QoL. Combination treatment with gemcitabine and nab-paclitaxel may provide significant benefit for advanced pancreatic cancer.

Trial registration: This study has been registered in ClinicalTrials.gov as NCT03807999 on January 8, 2019 (retrospectively registered).

Keywords: Nab-paclitaxel, Gemcitabine, Pancreatic cancer, Metastatic, Quality of life

\section{Background}

Approximately forty-thousand patients with pancreatic cancer die annually, which corresponds to $4^{\text {th }}$ most common cancer-caused deaths when both sexes combined. The incidence of pancreatic cancer has tripled since 1950s, nevertheless this sharp increase ranked pancreatic carcinoma only to the $10^{\text {th }}$ most common cancer regarding incidence rates. The significant difference between incidence and mortality rankings is associated with poor disease prognosis (mortality/incidence ratio: 98\%) [1]. The 5-year overall survival (OS) rate is approximately $4 \%$. Current evidence suggests that locally advanced or metastatic disease poorly responds to chemotherapy. When compared to fluorouracil, gemcitabine may modestly improve survival, but median OS in advanced cases still below 6 months [2]. Previous randomized phase-III studies showed no significant OS difference between cytotoxic drug combinations and gemcitabine-only regimens.

Randomized phase III PRODIGE trial evaluated FOLFIRINOX regimen in metastatic pancreatic cancer patients [3]. Both median progression-free survival (PFS) (6.4 vs 3.3 months, $p<0.001)$ and median OS (11.1 vs 6.8 months, $p<0.001$ ) were dramatically improved. In patients with good performance status FOLFIRINOX remains a viable first-line option. However, toxicity of FOLFIRINOX regimen still remains a concern.

The effect of FOLFIRINOX on quality of life (QoL) in metastatic pancreatic cancer was analyzed from the PRODIGE 4/ACCORD 11 trial [4]. FOLFIRINOX combination was found to significantly reduce QoL impairment compared with single-agent gemcitabine. Moreover, incorporation of baseline QoL scores to clinical and demographic data showed better survival probabilities.

The albumin-bound paclitaxel, namely nab-paclitaxel (nab-P), is a particular nanoparticle form of paclitaxel. The phase-III MPACT trial compared nab-P with gemcitabine in 861 patients with metastatic pancreatic adenocarcinoma. In this study, the nab-P arm received $125 \mathrm{mg} / \mathrm{m} 2$ of nab-p then $1000 \mathrm{mg} / \mathrm{m} 2$ gemcitabine for 3 weeks followed by a week of rest, and gemcitabine arm received $1000 \mathrm{mg} / \mathrm{m} 2$ of gemcitabine monotherapy for 7 weeks followed by a week of rest, and then weekly gemcitabine for 3 weeks plus 1 week of rest [5]. Authors reported that OS was significantly improved in nab-P arm (median $8.5 \mathrm{mo}$ ) compared to gemcitabine monotherapy (median $6.7 \mathrm{mo})(\mathrm{HR}=0.72$; $p<0.001$ ), which suggests a $31 \%$ reduction in the risk of progression or death. Twelve-month survival rates were $35 \%$ vs $22 \%$ in combination and gemcitabine-only regimens, respectively, which suggests a $59 \%$ increase in survival $(p=0.0002)$. Moreover, median PFSs were 5.5 vs 3.7 months (HR: $0.69 ; p=0.000024$ ), and overall response rates were $23 \%$ vs $7 \%$, respectively, which all favors combination treatment. The toxicity of the combination was modest and easily manageable. This combination may represent a new standard in the management of these patients.

QoL changes in patients receiving nab-P in combination with gemcitabine for the first-line treatment of metastatic or locally advanced unresectable pancreatic adenocarcinoma have not been explored. This randomized, phase II study analyzes the effect of nab-P plus gemcitabine on QoL of these patients. Efficacy and safety of the combination will also be analyzed. The randomized phase-III MPACT trial showed that gemcitabine + nab-P combination has superior clinical efficacy than gemcitabine-only regimen, but QoL associated with combination regimen at first-line in unresectable locally advanced or metastatic pancreatic ductal adenocarcinoma is still unknown.

\section{Methods}

This study included a total of 125 patients $\geq 18$ yearsold and presented with metastatic or unresectable pancreatic adenocarcinoma and without prior chemotherapy. Twenty-three patients (18.4\%) had locally advanced disease, and 102 patients $(81.6 \%)$ had metastatic disease. Other inclusion criteria were having a measurable/evaluable disease by RECIST, ECOG performance status 0 or 1 , adequate bone marrow functions (granulocyte count $\geq 1500 / \mathrm{mm}^{3}$, platelet count $\geq 100,000 /$ $\mathrm{mm}^{3}$ ), and adequate liver functions (Total biliribin $<2$ $\mathrm{mg} / \mathrm{dL}, \mathrm{ALP} / \mathrm{GGT}<5 \times$ upper normal limit - UNL, ALT/AST $<2.5 \quad x \quad$ UNL). The study protocol was 
approved by the Malatya Clinical Trials Ethical Committee of the Inonu University on 21 May 2014, and a signed informed consent were obtained from all patients. Exclusion criteria were as follows:

- Patients over 76 years-old, with active infection or chronic diarrhea

- Any prior treatment for metastatic pancreatic cancer. Only exception is systemic adjuvant treatment with/without radiation that completed $>6$ months before enrollment

- Being unable to comply to protocol

- Presence of severe cardiac disease including but not limited to congestive heart failure, symptomatic coronary artery disease, uncontrolled cardiac arrhythmias, or myocardial infarction within the last 12 months

- Presence of any other major organ failure, or metastases in central nervous system

- Expert opinion about increased risk of treatment or possibility of getting misleading results that might bias the study

- Very poor life expectancy that $<12$ weeks

- Pregnancy (positive pregnancy test) or lactation

- Prior malignancy other than skin cancer (basal cell), in-situ cervical cancer, any well-treated Stage-I/II cancer with complete remission or disease-free status more than 5 years

- Physically disintegrated upper gastro-intestinal tract, or presence of any malabsorption syndrome

- Uncontrolled coagulopathy, or concurrent/preexisting coumadine use

- Sensory neuropathy $>$ grade 1 .

- Major surgery without complete recovery within 4 weeks of the study commencement

Following recruitment, patients were 1:1 randomized to receive gemcitabine+nab-P, or gemcitabine alone. Treatment continued until disease progression or unacceptable toxicity.

Patients were treated on an outpatient basis with nab$\mathrm{P}+$ gemcitabine combination or single-agent gemcitabine. Patients receiving nab-P + gemcitabine received 30-40 min infusion of $125 \mathrm{mg} / \mathrm{m}^{2}$ nab-P ( $\max 40 \mathrm{~min}$ ) followed by $30-40 \mathrm{~min}$ infusion of $1000 \mathrm{mg} / \mathrm{m}^{2}$ gemcitabine ( $\max$ $40 \mathrm{~min}$ ) for 3 weeks, followed by a week of rest.

Patients receiving gemcitabine alone received weekly $30-40 \mathrm{~min}$ infusion of $1000 \mathrm{mg} / \mathrm{m}^{2}$ gemcitabine ( $\max 40$ min) for 7 weeks, followed by a week of rest (8-week cycle; Cycle 1 only), followed by cycles of weekly administration for 3 weeks (on Days 1, 8, and 15) followed by one week of rest (4-week cycle). Available therapies were given for the 2nd line treatment and supportive care on investigator's discretion.
Primary endpoint was 3-month deterioration free rate (percentage of patients free from definitive deterioration). The time until definitive deterioration (TUDD) was calculated using the time that an at least 10 point of decrease in EORTC QLQ-C30 scores has been observed. The quality of life assessments were also compared between study arms. The QLQ-C30 assessments were done every 4 weeks based on recommendations from EORTC.

Secondary endpoints were overall survival, progression free survival, and response rate.

\section{EORTC QLQ-C30 questionnaire}

The QoL of patients was assessed using the healthrelated quality of life questionnaire for cancer patients that developed by the European Organization for Research and Treatment (EORTC), the QLQ-C30 questionnaire. This scale included 30 items, which evaluate the symptoms and functions of the patients in 5 function domains as physical, role, social, emotional and cognitive functioning; 9 symptom domains as pain, fatigue, financial impact, appetite loss, nausea/vomiting, diarrhea, constipation, sleep disturbance; and, an overall QoL score that reflects the global health status.

\section{Statistical analysis}

Descriptive statistics were presented with mean or median for numerical variables, and frequency and percent for categorical variables. Regarding comparisons between treatment arms, the numerical data including EORTCQLQ-C30 scores were compared using Mann-Whitney $\mathrm{U}$ test, and the categorical data including proportions of patients with and without deterioration in QoL were compared using Chi-square test. Survival analyses regarding the TUDD were conducted with Kaplan-Meier method, and comparisons of survival curves were analyzed with log-rank test. SPSS 21 (IBM Inc., Armonk, NY, USA) software was used for the statistical analyses of the study.

\section{Results}

A total of 125 patients were included in the study. Median ages of the patients in gemcitabine arm were significantly younger than the patients in gemcitabine+nab-P arm $(p=0.031)$, but sex distribution were similar in both groups $\left(\mathrm{M} / \mathrm{F}_{\text {gemcitabin }}: 38 / 25 ; \mathrm{M} / \mathrm{F}_{\text {gemcitabine + nab-p: }}\right.$ $38 / 24 ; p=0.911$ ). Forty percent of disease localization in gemcitabine arm and $45 \%$ in gemcitabine+nab-P arm were at pancreatic head, and distributions of disease localization were similar between study arms $(p=0.325)$. Comparison of treatment response between arms revealed a significant difference $(p=0.009)$, which caused by the progressive disease in gemcitabine arm (42.4\%) versus gemcitabine+nab-P arm (19.4\%). General characteristics of patients were summarized in Table 1. 
Table 1 General demographics and clinical characteristics of study arms

\begin{tabular}{llll}
\hline & Gemcitabine & Gemcitabine + nab-P & $\mathbf{p}$ \\
& Median [range] & Median [range] & \\
Age (years) & $65.5[37-78]$ & $62[26-76]$ & 0.031 \\
& $\mathbf{n}(\%)$ & $\mathbf{n}(\%)$ & $\mathbf{p}$ \\
Sex & & & 0.911 \\
Male & $38(60.3)$ & $38(61.3)$ & \\
Female & $25(39.7)$ & $24(38.7)$ & 0.325 \\
Localization & & & \\
Head & $25(40.3)$ & $27(45)$ & \\
Head + corpus & $8(12.9)$ & $4(6.7)$ & \\
Corpus & $11(17.7)$ & $11(18.3)$ & \\
Corpus + tail & $5(8.1)$ & $7(11.7)$ & \\
Tail & $7(11.3)$ & $10(16.7)$ & \\
Response & & & \\
CR & $2(3.4)$ & - & \\
PR & $12(20.3)$ & $23(37.1)$ & \\
SD & $20(33.9)$ & $27(43.5)$ & \\
PD & $25(42.4)$ & $12(19.4)$ & \\
\hline
\end{tabular}

*Rate of progressive disease is responsible from the difference
Regarding the primary endpoint of the study, overall QoL analyses showed that 34 and $58.3 \%$ of cases in gemcitabine and gemcitabine+nab-P arms had no deterioration in 3rd month QoL scores $(p=0.018)$. These proportions were 27.3 and $36.6 \%$ in 6th month assessments, respectively $(p=0.357)$.

The QoL assessments are presented in Table 2. Percent changes in gemcitabine and gemcitabine+nab- $P$ arms at 3rd month assessments revealed that functional scales of EORTC-QLQ-C30 scale were significantly improved in gemcitabine+nab-P arm, whereas these were deteriorated in gemcitabine arm. The percent changes in the symptom scales were similar between study arms, but the fatigue score increased more in gemcitabine arm significantly, which is related with increased fatigue in patients receiving gemcitabine. The 6th month QoL assessments revealed that the percent changes in both study arms from the previous assessment were not statistically different. All patients completed the QoL questionnaires during treatment period without attrition, and there was no differential compliance between treatment arms that might affect the results of QoL assessments.

The median overall survival was 9.92 and 5.95 months in the combination and single-agent arms, respectively (HR: 0.642 , 95\% CI: 0.422 to $0.866, p=0.038$ ). Median PFS was 6.28 , and 3.22 months in the respective arms (HR: 0.582 , 95\% CI: $0.391-0.866, p=0.008$ ). The objective response rate was $37.1 \%$ vs $23.7 \%$, respectively, and

Table 2 QoL assessments at $3^{\text {rd }}$ and $6^{\text {th }}$ months

\begin{tabular}{|c|c|c|c|c|c|c|}
\hline & \multicolumn{3}{|c|}{$3^{\text {rd }}$ month QoL change $\%$} & \multicolumn{3}{|c|}{$6^{\text {th }}$ month QoL change $\%$} \\
\hline & Gemcitabine & Gemcitabine + nab-Paclitaxel & $p$ & Gemcitabine & Gemcitabine + nab-Paclitaxel & $p$ \\
\hline & Mean & Mean & & Mean & Mean & \\
\hline \multicolumn{7}{|l|}{ Functional scales } \\
\hline Cognitive functioning & -8.8 & 23.8 & 0.048 & 21 & 22.5 & 0.387 \\
\hline Emotional functioning & 12.9 & 50.8 & 0.048 & 0.6 & 37.8 & 0.851 \\
\hline Physical functioning & -11.7 & 12.8 & 0.051 & -4.9 & 5.7 & 0.761 \\
\hline Role functioning & 2.9 & 17.4 & 0.039 & 42.3 & 10.9 & 0.456 \\
\hline Social functioning & -1.6 & 30.4 & 0.024 & 7.5 & 3.9 & 0.743 \\
\hline \multicolumn{7}{|l|}{ Symptom scales } \\
\hline Appetite loss & -12.5 & -14.4 & 0.226 & -13.3 & -15 & 0.771 \\
\hline Constipation & 14.1 & 25.9 & 0.706 & 22.9 & 8.6 & 0.888 \\
\hline Diarrhea & 2.6 & 17.6 & 0.558 & 16.9 & -1.4 & 0.707 \\
\hline Dyspnea & -32.6 & -7.4 & 0.172 & 11 & -17.6 & 0.206 \\
\hline Fatigue & 60.4 & 5.9 & 0.027 & -51.4 & -30.4 & 0.924 \\
\hline Financial difficulties & -11 & 12.1 & 0.077 & -13.8 & 12.8 & 0.879 \\
\hline Insomnia & -19 & 11 & 0.141 & -9.6 & 7.7 & 0.276 \\
\hline Nausea-vomiting & -4.1 & 12 & 0.559 & -0.6 & -0.8 & 0.898 \\
\hline Pain & -13.8 & 12.4 & 0.142 & -11.3 & -16.6 & 0.514 \\
\hline Global health status/QoL & 12.7 & 8 & 0.163 & 70.6 & -9.2 & 0.158 \\
\hline
\end{tabular}


Table 3 Overall- and progression-free survival in study arms

\begin{tabular}{|c|c|c|c|c|c|c|}
\hline & Gemcitabine & Gemcitabine+nab-P & Overall & $H R^{*}$ & $95 \% \mathrm{Cl}$ & $p$ \\
\hline Median Overall Survival (mo) & 5.95 & 9.92 & 8.02 & 0.642 & $0.422-0.866$ & 0.038 \\
\hline Median Progression-free Survival (mo) & 3.22 & 6.28 & 4.60 & 0.582 & $0.391-0.866$ & 0.008 \\
\hline
\end{tabular}

*Age-adjusted HR for Gemcitabine vs. Gemcitabine + Nab-P arm

the difference was statistically significant $(p=0.009)$. And, median TUDD was 5.36 vs 3.68 months, respectively. The overall and progression-free survival in both arms are presented in Table 3 and Fig. 1.

\section{Discussion}

In this randomized, phase II study, we have evaluated the effects of nab-P + gemcitabine on QoL, at the firstline treatment of patients with unresectable locally advanced or metastatic pancreatic ductal adenocarcinoma. At the time of the study conducted, the nab-P was not registered and licensed for use in the Turkey, and this study has also provided an opportunity for the patients to reach to this treatment option. As an overall interpretation of our results, when compared with gemcitabine only, gemcitabine+nab-P was associated with an overall and progressive free survival advantage, with increased response rate, without increasing toxicity and deterioration of quality of life. Although these are promising results about treatment associated QoL of patients, one may still have concerns about including both unresectable locally advanced and metastatic patients in the analyses as a common group, which might be seen as a confounding factor to interpret the outcomes. But, since the proportion of unresectable locally advanced patients are less than one fifth of the total population, and since both groups share a common approach regarding treatment in our study, we have not separated the analyses, and think that our results are more generalizable to the treatment of pancreatic cancer patients with advanced disease.

The trajectory of the treatment of advanced pancreatic cancer showed significant survival advantage during its course, but not without sacrificing the QoL of the patients. The primary objective of drug development is to improve patient survival, and this applies to the clinical trials in advanced pancreatic cancer. In line with this aim, first effective treatment for pancreatic cancer has emerged as gemcitabine in 1997 [2]. In 2011, PRODIGE trial revealed that FOLFIRINOX provides significant overall and progression-free survival advantage [3]. And, recently MPACT trial reported that nab-P + gemcitabine combination for the first-line treatment of advanced pancreatic cancer provided promising results as improved overall and progression-free survival in these patients [5]. Currently, among the available therapeutic options for advances pancreatic cancer, data about the QoL of these patients is only available for the FOLFIRINOX regimen, which suggests a deterioration in health-related QoL of these patients [4]. The authors also reported that incorporation of baseline QoL of patients for prediction of survival has prognostic value, which might be used as a stratification factor in further clinical trials. Nevertheless, QoL of patients with advanced pancreatic cancer is not currently being evaluated for treatment decision. Since recent advances in the treatment of these patient group has changed the approach to treatment decision by favoring nab-P + gemcitabine combination, the need for data about
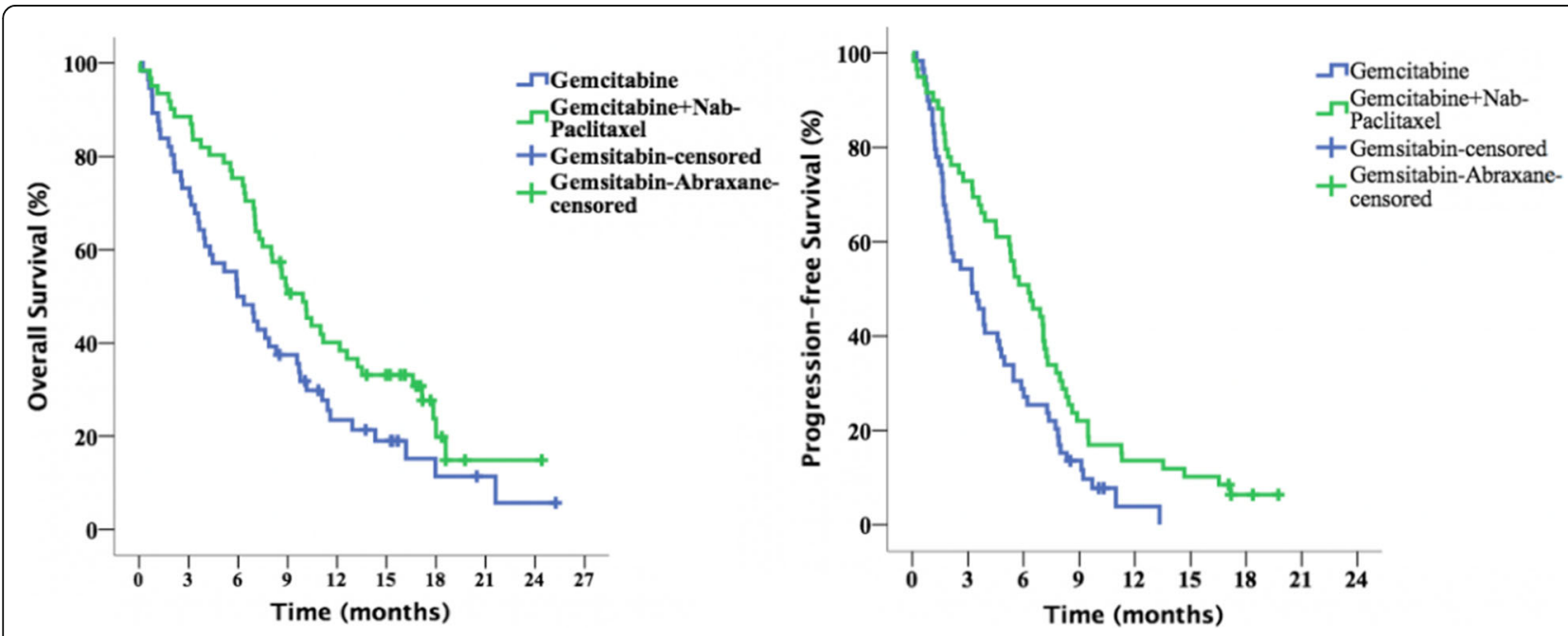

Fig. 1 Overall and progression-free survival 
the QoL during this treatment has emerged. To the best of our knowledge, this is the first study that evaluated QoL in first-line treatment of advanced pancreatic cancer with Abraxane+Gemcitabine. The rationale behind selecting the EORTC QLQ-C30 scale in this study is about providing a comparable data about the QoL of advanced pancreatic cancer patients with the reference paper, the PRODIGE trial, to infer about the probable QoL benefits of gemcitabine or combination with nab-P.

A recent systematic review has evaluated the effects of chemotherapy on QoL of patients with advanced pancreatic cancer [6]. According to the results of this review, 19 of 23 studies that evaluated the QoL did not report any difference between treatment arms that vary between studies selected. But, 4 studies have reported QoL of the patients with advanced pancreatic cancer showed significant difference between treatment arms. Accordingly, in EORTC QLQ-C30 assessments, global health scores, functional domains including physical, cognitive and role scores, and symptom domains including fatigue scores were found to be better in gemcitabine when compared to BAY12-9566 [7]. In another study that used Functional Assessment of Cancer Therapy-Pancreas (FACT-Pa) QoL questionnaire, gemcitabine and placebo was better than gemcitabine-marimastat combination [8]. Third study revealed that FOLFIRINOX provided favorable outcomes in EORTC QLQ-C30 assessments when compared to gemcitabine, which was reported as definitive degradation of QoL of $31 \%$ vs $66 \%$, respectively (HR: $0.47,95 \%$ CI: 0.30 to $0.70, P<0.001)[3]$. And the last study revealed that fluorouracil + cisplatin combination provided better QoL outcomes measured by Spitzer's Quality of Life Index than fluorouracil alone [9]. As can be seen from these studies, one cannot exactly comment on a specific chemotherapeutic agent to provide favorable QoL outcomes than the other agents. Nevertheless, the agents assessed in above studies have some gradual survival advantages to each other, which lacks to be supported by the QoL advantage.

One of the main reasons for heterogeneity in the results of comparison trials about QoL in advanced pancreatic cancer is mainly based on the nature of the disease. Besides the disease itself possess a significant burden of mortality and morbidity on patients, it also causes distressing symptom, which the two most common are pain and cachexia $[10,11]$. These two primary factors that tightly associated with pancreatic cancer also has significant contribution to the deterioration of QoL. The variability in the presence of these clinical conditions affect the results of trials that compare the QoL between treatment agents. Since these major symptoms and QoL of the patients are significantly correlated, controlling for the QoL at treatment initiation might be an alternative approach for treatment decision. Moreover, close follow-up of patients for QoL during treatment and appropriate interventions to improve QoL might provide additional advantage for the patient outcomes in advanced pancreatic cancer treatment.

Secondary outcomes in this study were the overall and progression-free survival. Our results revealed that nab$\mathrm{P}+$ gemcitabine arm had significant overall (9.92 vs. 5.95 mo.) and progression-free (6.28 vs. 3.22 mo.) survival advantage when compared to gemcitabine alone. In previous randomized studies that compared nab-P + gemcitabine vs. gemcitabine alone in advanced pancreatic cancer, OS advantage was 2.1 months (median 8.7 vs. 6.6 months) in MPACT trial and 4.8 months (median 11.9 vs. 7.1 months) in the Canadian subgroup analysis of MPACT trial; and PFS advantages were 1.8 months (median 5.5 vs. 3.7 months) and 2 months (median 5.5 vs. 3.7 months) in corresponding studies, respectively. The 4 months of OS advantage and 3 months of PFS advantage in our study are comparable with these previous reports, which suggest similar efficiency of nab-P + gemcitabine in patients with advanced pancreatic cancer. The contribution of QoL advantage in this treatment option also contributes much to the data about the outcomes of this treatment regimen.

\section{Conclusions}

As an overall conclusion of our study, gemcitabine and nab-P combination regimen is a preferable option for the treatment of patients with advanced pancreatic cancer when compared to gemcitabine alone, by means of both survival advantage and also deterioration-free QoL.

\section{Abbreviations \\ ALP: Alkaline phosphatase; ALT: Amino alanine transferase; AST: Aspartate amino transferase; Cl: Confidence interval; ECOG: Eastern Cooperative Oncology Group; EORTC: European Organisation for Research and Treatment of Cancer; FACT-Pa: Functional Assessment of Cancer Therapy-Pancreas; GGT: Gamma-glutamyl transpeptidase; nab-P: Nab-paclitaxel; M/F: Male/ Female; M/I ratio: Mortality/Incidence ratio; MPACT: Metastatic Pancreatic Adenocarcinoma Clinical Trial; OS: Overall survival; PFS: Progression-free survival; QoL: Quality of life; RECIST: Response Evaluation Criteria In Solid Tumors; TUDD: Time Until Definitive Deterioration; UNL: Upper normal limit}

\section{Acknowledgements}

This study has been presented as a poster in Gastrointestinal Cancers Symposium of American Society of Clinical Oncology (ASCO) in 2018, and the abstract has been published in Journal of Clinical Oncology (JCO) 36, no. 4_suppl (February 01, 2018) 346-346 [12].

\section{Authors' contributions}

The primary author of the study, SY, has designed the study and contributed to data collection, interpretation of the results of analyses, manuscript preparation. The authors, FD, BO, NOY, Al, MO, GD, HSC, BK, TE, MAU, FO, HT, TY, FA, AS, HA, and SK, were responsible for data collection. DY and $\mathrm{MH}$ have conducted the statistical analyses, and contributed to interpretation of the results of analyses. DY has also contributed to manuscript preparation. All authors read and approved the final version of the manuscript.

\section{Funding}

This study was sponsored by the primary investigator (SY). Also, study medication and costs of medical tests were covered by the pharmaceutical company (Celgene) manufacturing the study drug. The external funding 
body (Celgene) had no role in design of the study and collection, analysis, and interpretation of data and in writing the manuscript.

\section{Availability of data and materials}

The datasets used and/or analyzed during the current study are available from the corresponding author on reasonable request.

\section{Ethics approval and consent to participate}

This study was approved by the Malatya Clinical Trials Ethics Committee of Inonu University. Approval date was 21 May 2014, and approval number was 2014/58.

Written informed consent was obtained from all patients.

\section{Consent for publication}

Not applicable

\section{Competing interests}

SY: Honorarium due to advisory board activity, speaker, bureau activity from Roche, Novartis, Amgen, Gen llac, Merck Serono, Pfizer, Lilly, Sanofi, MSD, BMS, Teva, Celgene Abbott, and Bayer.

\section{Author details}

${ }^{1}$ Hacettepe University Faculty of Medicine, Ankara, Turkey. ${ }^{2}$ Hacettepe University Cancer Institute, Ankara, Turkey. ${ }^{3}$ Marmara University Faculty of Medicine, İstanbul, Turkey. ${ }^{4}$ Dr. Abdurrahman Yurtaslan Ankara Oncology Training and Research Hospital, Ankara, Turkey. ${ }^{5}$ Ankara Numune Training and Research Hospital, Ankara, Turkey. ${ }^{6}$ Dicle University Faculty of Medicine, Dyarbakir, Turkey. ${ }^{7}$ Erciyes University Faculty of Medicine, Kayseri, Turkey. ${ }^{8}$ Ondokuz Mayis University Faculty of Medicine, Samsun, Turkey. ${ }^{9}$ Akdeniz University Faculty of Medicine, Antalya, Turkey. ${ }^{10}$ Ege University Faculty of Medicine, İzmir, Turkey. ${ }^{11}$ Uludag University Faculty of Medicine, Bursa, Turkey. ${ }^{12}$ Lütfi Kirdar Kartal Training and Research Hospital, İstanbul, Turkey. ${ }^{13}$ Karadeniz Teknik University Faculty of Medicine, Trabzon, Turkey. ${ }^{14}$ istanbul University Cerrahpasa Faculty of Medicine, Bursa, Turkey. ${ }^{15}$ Dokuz Eylül University Faculty of Medicine, İzmir, Turkey. ${ }^{16}$ Istanbul University Cancer Institute, İstanbul, Turkey. ${ }^{17}$ Gaziantep University Faculty of Medicine, Gaziantep, Turkey. ${ }^{18}$ Ankara University Faculty of Medicine, Ankara, Turkey.

Received: 16 March 2019 Accepted: 17 March 2020

Published online: 30 March 2020

\section{References}

1. Siegel R, Naishadham D, Jemal A. Cancer statistics 2013. CA: A Cancer J Clin. 2013;63:11-30.

2. Burris HA 3rd. Recent updates on the role of chemotherapy in pancreatic cancer. Semin Oncol. 2005;32(4 Suppl 6):S1-3.

3. Conroy $T$, Desseigne F, Ychou M, Bouché O, Guimbaud R, Bécouarn Y, Adenis A, Raoul JL, Gourgou-Bourgade S, de la Fouchardière C, Bennouna J, Bachet JB, Khemissa-Akouz F, Péré-Vergé D, Delbaldo C, Assenat E, Chauffert B, Michel P, Montoto-Grillot C, Ducreux M, Groupe Tumeurs Digestives of Unicancer; PRODIGE Intergroup. FOLFIRINOX versus gemcitabine for metastatic pancreatic cancer. N Engl J Med. 2011;364:1817-25.

4. Gourgou-Bourgade S, et al. Impact of FOLFIRINOX compared with gemcitabine on quality of life in patients with metastatic pancreatic cancer: results from the PRODIGE 4/ACCORD 11 randomized trial. J Clin Oncol. 2013;31(1):23-9.

5. Von Hoff DD, Ervin T, Arena FP, et al. Randomized phase III study of weekly nab- paclitaxel plus gemcitabine vs gemcitabine alone in patients with metastatic adenocarcinoma of the pancreas (MPACT) [oral presentation]. San Francisco, CA, USA: Gastrointestinal Cancers Symposium (ASCO); 2013; January 24-26.

6. Kristensen A, et al. Does chemotherapy improve health-related quality of life in advanced pancreatic cancer? A systematic review. Crit Rev Oncol Hematol. 2016:99:286-98

7. Moore MJ, Hamm J, Dancey J, Eisenberg PD, Dagenais M, Fields A, et al. Comparison of gemcitabine versus the matrix metalloproteinase inhibitor BAY 12-9566 in patients with advanced or metastatic adenocarcinoma of the pancreas: a phase III trial of the National Cancer Institute of Canada clinical trials group. J Clin Oncol. 2003;21:3296-302.

8. Bramhall SR, Schulz J, Nemunaitis J, Brown PD, Baillet M, Buckels JA. A double-blind placebo-controlled, randomised study comparing gemcitabine and marimastat with gemcitabine and placebo as first line therapy in patients with advanced pancreatic cancer. Br J Cancer. 2002;87:161-7.

9. Ducreux M, Rougier P, Pignon JP, Douillard JY, Seitz JF, Bugat $R$, et al. A randomised trial comparing 5-FU with 5-FU plus cisplatin in advanced pancreatic carcinoma. Ann Oncol. 2002;13:1185-91.

10. Labori KJ, Hjermstad MJ, Wester T, Buanes T, Loge JH. Symptom profiles and palliative care in advanced pancreatic cancer: a prospective study. Support. Care Cancer. 2006;14:1126-33.

11. Muller-Nordhorn J, Roll S, Bohmig M, Nocon M, Reich A, Braun C, et al. Health-related quality of life in patients with pancreatic cancer. Digestion. 2006;74:118-25.

12. Quality of life study in patients with unresectable locally advanced or metastatic pancreatic cancer treated with gemcitabine in combination with nab-paclitaxel versus gemcitabine alone:ax-panc-sy001: A phase ॥ randomized study. Journal of Clinical Oncology 36, no. 4 suppl (February 01, 2018) 346-346. doi:https://doi.org/10.1200/JCO.2018.36.4_suppl.346.

\section{Publisher's Note}

Springer Nature remains neutral with regard to jurisdictional claims in published maps and institutional affiliations.
Ready to submit your research? Choose BMC and benefit from:

- fast, convenient online submission

- thorough peer review by experienced researchers in your field

- rapid publication on acceptance

- support for research data, including large and complex data types

- gold Open Access which fosters wider collaboration and increased citations

- maximum visibility for your research: over $100 \mathrm{M}$ website views per year

At BMC, research is always in progress.

Learn more biomedcentral.com/submissions 